\title{
'Soltanto le montagne non si incontrano'. Buone pratiche per il recupero di colture/culture locali fra tradizione e innovazione
}

"Only the mountains do not meet". Good practices for the recovery of local crops/cultures between tradition and innovation

\section{Laura Bonato}

\section{OpenEdition Journals}

Edizione digitale

URL: https://journals.openedition.org/aam/4580

DOI: $10.4000 /$ aam.4580

ISSN: 2038-3215

\section{Editore}

Dipartimento Culture e Società - Università di Palermo

\section{Notizia bibliografica digitale}

Laura Bonato, «'Soltanto le montagne non si incontrano'. Buone pratiche per il recupero di colture/ culture locali fra tradizione e innovazione», Archivio antropologico mediterraneo [Online], Anno XXIV, n 23 (2) | 2021, online dal 31 décembre 2021, consultato il 08 janvier 2022. URL: http:// journals.openedition.org/aam/4580 ; DOl: https://doi.org/10.4000/aam.4580

Questo documento è stato generato automaticamente il 8 janvier 2022.

\section{c) (†)}

Archivio antropologico mediterraneo è distribuita con Licenza Creative Commons Attribuzione - Non commerciale - Non opere derivate 4.0 Internazionale. 


\title{
'Soltanto le montagne non si incontrano' ${ }^{1}$. Buone pratiche per il recupero di colture/culture locali fra tradizione e innovazione
}

\author{
"Only the mountains do not meet". Good practices for the recovery of local \\ crops/cultures between tradition and innovation
}

Laura Bonato

\section{Premessa}

La sintetica agenda delle attività e dei progetti a favore della ripresa di colture abbandonate - nello specifico la segale - nelle pagine che seguono sembra confermare una tendenza degli ultimi anni che le scienze sociali stanno documentando e analizzando, cioè quella del "ritorno alla terra", nella sua doppia accezione di ri-abitare gli spazi alpini e di dedicarsi a mestieri dimenticati. In molti casi questo fenomeno - di particolare significatività - assume un valore ideale, che identifica la montagna come "nuovo" orizzonte per il futuro, a scapito della "cattiva" città generatrice di crisi. Gli esiti della personale esperienza di campo - da cui nasce questo contributo suggeriscono che ripristinare colture abbandonate pare una risposta possibile all'attuale crisi: rientrare in un sistema semplice, come quello agropastorale, è una scelta che sembra essere condivisa da più persone, soprattutto giovani. A fronte di un sistema produttivo ed economico basato sul principio di crescita illimitata dei consumi e del PIL, ottiene sempre più consensi una prospettiva economico-sociale alternativa, dal punto di vista anche puramente esistenziale, teorizzata da Serge Latouche (2008): la decrescita, una "filosofia" che prevede un abbassamento del PIL e che è incentrata sul miglioramento del "ben-essere", a scapito del "ben-avere", privilegiando aspetti extraeconomici e spesso dimenticati, quali la cultura o le relazioni umane: «la crescita economica senza limiti, considerata come fine a se stessa, ha portato l'uomo a 
rinunciare ogni giorno di più alla sua parte di umanità. [...] L'ipermaterialismo che ne è derivato ha condotto la specie umana nell'impasse di una miseria psichica e morale» (Besson-Girard 2007: 27). La decrescita non è da intendersi come crescita negativa ma come affrancamento dal mito economico della crescita, riscoprendo "antichi" valori e riorganizzando il paradigma produttivo e sociale. Questa filosofia è fortemente condivisa dal movimento neorurale, un fenomeno sociale di ritorno alla terra nella sua dimensione più intima e biologico-produttiva, «una delle tendenze socio-culturali più caratteristiche della post-modernità, fenomeno legato alla crisi dell'urbanesimo occidentale, reazione al degrado ecologico e sociale della città moderna» (Salsa 2007: 116). I suoi sostenitori sono in primis giovani con esperienze lavorative diverse da quelle agricole o che, dopo aver conseguito un titolo di studio, decidono di coltivare la terra stimolati da un significativo interesse nei confronti di temi quali autosostenibilità, agricoltura biologica, rispetto per l'ambiente ${ }^{2}$. Nel mondo rurale, lo stesso abbandonato e rifuggito dalla generazione precedente, questi giovani individuano valide opportunità lavorative ed economiche ma anche un modo per migliorare la qualità della propria vita. Il ritorno all'agricoltura in montagna rientra quindi in un'idea socio-economica di sostenibilità, principalmente ambientale, che a sua volta è collegata al concetto di resilienza che, al di là della capacità umana di reagire positivamente alle sollecitazioni negative, è qui da intendersi come adozione di uno stile di vita attento all'ambiente e flessibile al cambiamento.

\section{Contiguità}

Nel nostro paese a partire dagli anni Sessanta-Settanta del secolo scorso, di contro ai macroscopici processi di industrializzazione e di urbanizzazione, si assiste ad iniziative comunitarie di riscoperta, recupero e riattivazione di elementi della tradizione agropastorale e locale; sono sostanzialmente due gli aspetti di tale tradizione interessati: la ritualità e il lavoro contadino e artigiano, i cui attrezzi e prodotti vengono conservati nei molti musei locali appositamente creati. I protagonisti, gli esecutori, coloro che manifestano questi tratti di cultura il più delle volte non sono portatori ingenui e immediati, che hanno ricevuto oralmente o per osservazione quotidiana le relative competenze e nozioni; sono invece interpreti più consapevoli, non di rado esponenti di associazioni locali, che li hanno assunti in modo intenzionale e mediato, anche con l'intervista e la consultazione di membri più anziani della comunità, $\mathrm{o}$ con l'imitazione di comunità vicine e talora addirittura di eventi comunicati dai media. Le intenzioni, le finalità di questi protagonisti e interpreti della tradizione possono essere ricondotte ad un iniziale tentativo di salvaguardare dall'impatto dello sviluppo industriale e urbano certi aspetti della memoria e dell'identità locale, che col tempo vengono sempre più spesso concepiti anche - o principalmente - come risorse per lo sviluppo del territorio, per la promozione delle produzioni locali, per la fruizione del paesaggio, per il turismo. Questa "imprenditorialità culturale" rivela che all'intento di tutela delle tradizioni si è aggiunta la consapevolezza che aspetti della memoria, elementi della quotidianità e la ritualità del passato possono diventare una forma di comunicazione rivolta all'esterno della comunità locale, un'offerta turistica di supporto alla sua economia ${ }^{3}$. Questi tratti di cultura non sono più soltanto argomento di ricerca scientifica, storica, archeologica, di catalogazione e lavoro documentario, e poi di fruizione elitaria, ma si configurano sempre più come oggetto di ricezione e utilizzo di massa e si presentano come 
potenzialità unica per lo sviluppo di singole zone; fanno parte della vita di una comunità, la quale li produce e li utilizza come strumento di espressione di se stessa. È opportuno soffermarsi sul concetto di comunità per specificare che sarà qui considerata «sia come oggetto e contesto di indagine sia come soggetto che agisce sul territorio» e i suoi membri utilizzano «strategie diverse per affermare il loro senso di appartenenza e il legame con il contesto in cui hanno scelto di vivere» (Porcellana, Stefani 2016: 10). Le comunità locali sono suscettibili alla variazione, si riconfigurano rapidamente, cambiano per l'apporto che nuovi membri possono offrire, per l'introduzione o l'eliminazione di elementi fisici del territorio ma anche, e soprattutto, perché variano le modalità con le quali gli individui si rapportano al proprio spazio di vita e gli attribuiscono valore. Azioni comuni di sviluppo, progetti di ricerca, tutela e salvaguardia relativi al patrimonio culturale, nel tentativo di conciliare tradizione e innovazione, sempre più documentano nuove pratiche sostenibili e propongono soluzioni innovative e - appunto - sostenibili per la valorizzazione dei beni culturali. Sostenibilità sembra essere la parola chiave affinché la cultura si mantenga vitale, accessibile, dinamica, equa ${ }^{4}$.

Sostenibilità è un termine che rientra nel linguaggio ricorrente, ampiamente usato, forse persino abusato; in particolare lo impieghiamo per valutare aree territoriali e programmi di sviluppo. Indubbiamente quello di "sostenibilità" è un concetto particolarmente complesso, dinamico e che si è evoluto negli anni: è stato introdotto nel 1972, nell'ambito della prima conferenza ONU sull'ambiente, incentrato prevalentemente sulla dimensione ecologica, è poi «entrato stabilmente nel vocabolario dello sviluppo a partire dal Rapporto Brundtland della World Commission on Environment and Development (1987)» (Colajanni 2008: 109), per acquisire nel tempo una valenza più ampia, che tiene conto dell'aspetto ambientale (responsabilità nell'uso delle risorse), economico (capacità di generare reddito e occupazione) e sociale (sicurezza, salute, ricchezza), come pure delle «capacità del contesto locale di generare produttività adeguata, lavoro, capacità organizzative e know how, con proprie risorse, in modo da produrre lentamente una situazione di non-dipendenza dagli investimenti esterni per lo sviluppo» (Colajanni 2008: 109). L'attenzione al contesto sociale «come variabile fondamentale nei processi di sviluppo» si rapporta ad una sostenibilità di tipo sociale, attributo facilmente estendibile a socioculturale, in quanto «ogni gruppo umano, ogni comunità [...] possiede [...] idee, concezioni di vita, credenze e valori» (Colajanni 2008: 110). Lo sviluppo sostenibile si definisce dunque come il prodotto di quelle attività umane che usano le risorse di determinati territori per incrementare la qualità della vita. Ma la nozione stessa di sviluppo prelude ad una crescita illimitata, la quale, però, diventa sostenibile solo se viene limitata per rispettare i vincoli posti dalla natura (Colajanni, Giancristofaro, Sacco 2020). Lo sviluppo può essere considerato sostenibile se incontra i bisogni dell'attuale popolazione locale senza compromettere quelli delle generazioni future. Sostenibilità significa, in sostanza, benessere, costante e in crescita: e questo "potenziale di crescita" si può scoprire attraverso elementi strategici come l'ambiente e l'agricoltura, per consentire un idoneo sviluppo economico delle comunità locali anche grazie alla tutela dei paesaggi agrari e dei patrimoni culturali. In termini di sviluppo rurale, l'innovazione territoriale deve essere considerata e associata allo sviluppo sostenibile, con la riorganizzazione e la messa in comune dei territori e con il rafforzamento dei network di comunicazione che possono portare ad una competitività territoriale e ad uno sviluppo economico (Mancini 2015). Saranno quindi qui indagate nuove pratiche, osservabili da qualche anno in alcune aree 
- in particolare quelle riconosciute come "marginali" -, sorte nell'ottica di una crescita più equilibrata e sostenibile che sta generando processi virtuosi di sviluppo locale. $\mathrm{Ne}$ conseguono, come si vedrà, nuovi modi creativi di porsi nei confronti dei territori interni, in contesti di vita periferici e di piccole dimensioni, per quanto riguarda il fare impresa, l'utilizzo delle risorse locali e la fruizione ambientale, oltre che l'abitare. Un caso esemplare, in questo senso, è la nascita, negli ultimi anni, di associazioni e aziende agricole che stanno cercando di reintrodurre sul territorio colture storicamente documentate ma scomparse, attivando nel contempo processi innovativi di produzione culturale e di creazione di socialità.

Negli ultimi anni le scienze sociali e le discipline affini hanno documentato, analizzato e riflettuto sul fenomeno del "ritorno alla terra" nella sua doppia accezione di ri-abitare gli spazi e di dedicarsi a lavori abbandonati, come la coltivazione di determinate specie vegetali o l'allevamento. In molti casi questo ritorno assume un valore ideale: si configura come la speranza - o l'illusione - che le zone rurali possano costituire un "nuovo" orizzonte per il futuro. In quest'ottica si inseriscono alcuni progetti di cui sono - o sono stata di recente - coordinatrice. Il progetto M.A.S.K.A. - Marginal Areas. Sustainability and Know-how in the Alps -, finanziato dalla Compagnia di San Paolo (2015-2017), si poneva l'obiettivo di documentare, osservare e sostenere attraverso interventi concreti il processo di riconversione delle terre marginali in aree produttive, rendendo possibile il riutilizzo di terre incolte (o che hanno subito l'avanzata della zona boschiva) e riattivando la filiera sia economica sia culturale e territoriale. La necessità primaria era facilitare nelle Alpi occidentali la promozione di nuove attività economiche che si distinguono per il loro potenziale sostenibile, attraverso azioni determinate e precise che coinvolgessero gli abitanti (Bonato 2017). Le aree marginali sono quelle realtà culturali e paesaggistiche che a causa del progressivo abbandono dell'agricoltura e dell'allevamento, e di conseguenza dello spopolamento, sono state lasciate incolte, provocando diversi effetti: dal rinselvaticamento, e quindi dal prosperare di zone boschive dove precedentemente esistevano quelle atte alla coltura, all'impoverimento del suolo. Il progetto Growing Consciousness (2020 e 2021), finanziato da EIT Food - European Institute of Innovation and Technology (EIT), un organismo indipendente dell'Unione Europea che stimola l'innovazione e l'imprenditorialità in tutta Europa -, mira a riattivare le catene agro-alimentari nelle aree rurali attraverso l'innovazione sostenibile. In particolare, con l'ausilio di una serie di attività che si muovono in un ambito di biodiversità agricola, cerca di produrre e promuovere abilità, tecniche e saperi volti a rivitalizzare in modo sostenibile la coltivazione di varietà di cereali, piante medicinali ed erbe aromatiche antiche e/o abbandonate nelle aree rurali dei paesi RIS (Regional Innovation Scheme) dell'Europa meridionale e orientale, cioè quelli in cui la tecnologia non è sviluppata come nei territori circostanti. Gli obiettivi del progetto sono sostenere e sperimentare coltivazioni che favoriscono un circolo di coltivazione-uso-divulgazione-innovazione; valutare criticamente l'impatto del cibo su ambiente, cambiamenti climatici e salute, in particolare per le comunità locali; incrementare la conoscenza delle buone pratiche coinvolgendo diverse parti interessate.

Un esempio significativo di "presa in cura" territoriale ${ }^{5}$, che dialoga contemporaneamente con la percezione della qualità della vita da parte degli abitanti $\mathrm{e}$ con le pratiche minute di vita sociale e culturale, è la ripresa della coltura della segale sul versante italiano dell'arco alpino, luogo privilegiato da secoli per la coltivazione di cereali e altri semi che un tempo costituivano non solo una fonte di sussistenza 
primaria ma anche un valido presidio per la cura delle affezioni più disparate, per uso interno ed esterno, rappresentando spesso anche degli elementi rituali nell'aspetto magico-religioso della cura.

\section{Adattabilità}

La segale, pianta della famiglia delle Graminacee, è il cereale di montagna per eccellenza, pur non essendo originaria delle $\mathrm{Alpi}^{6}$, data la sua resistenza al freddo: tra tutti i cereali è quello che germoglia alla temperatura più bassa, anzi predilige climi freddi - cresce fino a 4mila metri di altitudine - e si adatta a terreni difficili, poveri e sabbiosi; è poco esigente relativamente a concimazioni e pesticidi. Dal punto di vista botanico assomiglia al frumento, dal quale però si distingue per la maggiore altezza del fusto (Saltini 2020). Ben più resistente del grano, nelle Alpi e in quasi tutta l'Europa centrale la segale era coltivata fin dall'antichità e la sua diffusione è attestata dalle frequenti epidemie di ergotismo causate dall'ingestione di farina contaminata da segale cornuta - claviceps purpurea ${ }^{7}$-, «un fungo che, favorito da primavere e estati piovose, s'installa sui cereali [...] coprendoli di escrescenze nerastre dette sclerozi» (Ginzburg 1989: 284). Questa intossicazione prodotta dagli alcaloidi insorgeva in due forme, spesso entrambe mortali: cancrenosa, che si manifestava con disturbi circolatori agli arti fino alla cancrena delle estremità; e convulsiva, che provocava «crampi violentissimi, stati simili all'epilessia con perdita dei sensi per la durata di sei-otto ore ${ }^{8} 》$ (Ginzburg 1989: 284). Solo nel corso del ' 600 ne è stata scoperta la causa (Barger 1931). La medicina popolare impiegava la segale cornuta come abortivo; le donne la usavano per provocare dolori all'utero e come antiemorragico; le levatrici se ne servivano per accelerare le doglie, e quindi il parto: tutto ciò suggerisce che alcune sue proprietà erano note $\mathrm{e}$ controllate ma non è certo che se ne conoscessero le facoltà allucinatorie, anche se alcune denominazioni come «il francese seigle ivre (segale ubriaca) e il tedesco Tollkorn (grano pazzo) [...] sembrano indicare un'antica consapevolezza del potere racchiuso nella pianta» (Ginzburg 1989: 285).

La segale è stata indispensabile e fondamentale per l'alimentazione e per la quotidianità fino agli anni ' 50 del secolo scorso: pane; paglia per la lettiera degli animali; ottimo materiale, isolante e resistente, per la costruzione dei tetti. Esistono due tipologie di segale: invernale ed estiva. Nell'Europa centrale viene coltivata quasi esclusivamente la segale invernale, che può sfruttare meglio l'umidità e resiste ad un'eventuale siccità primaverile; quella estiva è tipica solo delle regioni in cui possono verificarsi gelate tardive e che si trovano in posizioni montuose esposte. Viene seminata in settembre/ottobre e raccolta in primavera (Wong 1998). Non essendo semplice da raccogliere né particolarmente produttiva, nel nostro paese la sua coltivazione si è gradualmente ridotta, fino quasi a scomparire. Per molto tempo questo cereale è stato sottovalutato e considerato un alimento povero, consumato dalle classi meno agiate in sostituzione/mancanza del frumento. Negli ultimi anni, però, la segale è stata riscoperta e, se pur alcuni casi di studio - si vedano ad esempio Grasseni (2013) e Orlando (2018) - mostrano che la ricerca del cibo "naturale" non procede necessariamente in parallelo con scelte etiche o con l'attenzione all'ambiente, nei contesti che ho potuto esplorare sembra andare di pari passo con il rinnovato interesse di molti consumatori per i cereali minori, la sempre più accentuata attenzione per gli acquisti a km zero e per un'alimentazione quanto più naturale e salutista, la crescente 
domanda di prodotti genuini, biologici a filiera corta, coltivati nel rispetto dell'ambiente e delle persone. Questo cereale è molto apprezzato e può rappresentare una risorsa importante per l'economia perché se ne possono ricavare prodotti alimentari, che rispondono ai richiesti sopraccitati canoni biologici e salutisti, e materiali isolanti destinati alla bioedilizia, che si fonda su abitazioni sostenibili e di basso impatto ambientale.

Il recupero e la reintroduzione della segale, che possiede proprietà nutrizionali $\mathrm{e}$ medicinali ${ }^{9}$ eccellenti, è indubbiamente interessante in un'ottica di biodiversità e di cibo biologico e sostenibile. Nello specifico, ora con quali modalità e quali intenti si sta recuperando questa coltura? Si tratta di un fenomeno di costume, che implica anche un rifiuto all'omologazione alimentare, o ha obiettivi ambientali e ricadute economiche non trascurabili? E qual è il coinvolgimento e il ruolo delle comunità locali? Nel tentativo di rispondere a tali quesiti, saranno inoltre qui analizzate questioni strettamente correlate relative al recupero di tale coltura in rapporto alla conservazione dell'ambiente attraverso la patrimonializzazione delle risorse disponibili sul territorio e al turismo del luogo. Le comunità locali, sempre più consapevoli della loro agency, affermano di investire le loro energie nel tentativo di non perdere le proprie usanze: queste, a mio parere, sono inserite in un tessuto relazionale politico, sociale, economico dal cui centro emergono figure che se ne fanno interpreti, quasi degli "imprenditori" - come accennato in apertura - delle tradizioni, rivitalizzando le quali determinano rilevanti trasformazioni socio-culturali ed economiche nel tessuto sociale. La riproposta e la patrimonializzazione di un bene sono infatti frutto di una progettualità elaborata: la "messa in scena" della tradizione permette ai locali non solo di perpetuare usi e pratiche di un tempo ma anche di manifestare la loro appartenenza alla comunità; non è quindi il passato ad essere rappresentato ma la comunità stessa, che si ricompone attraverso le iniziative che mirano alla tutela e alla conservazione.

\section{Spontaneità}

Nella sala conferenze del Centre d'Études francoprovençales di Saint-Nicolas, in Valle d'Aosta, c'è un bassorilievo ${ }^{10}$ che raffigura tutto il ciclo della coltivazione della segale fino alla panificazione. Questo artefatto può essere interpretato come espressione dell'immaginario della comunità locale perché in vari contesti analizzati ho riscontrato che quando si dice segale si pensa pane, quello nero, che in passato nell'arco alpino si preparava una volta all'anno, più precisamente all'inizio dell'inverno, quando i ritmi del lavoro contadino e le attività agropastorali rallentavano; una volta impastati, i pani venivano incisi con specifici segni di riconoscimento per poterli suddividere poi tra le varie famiglie e si procedeva alla cottura collettiva. Il pane nero veniva essiccato nei solai e conservato per tutto l'anno; lo si sarebbe poi consumato secco o ammorbidito nell'acqua, nel brodo, nel latte o nel vino. Dal 2015 circa 50 comuni valdostani hanno ripreso questo momento, divenuto ora di convivialità, e all'inizio dell'inverno si prepara il pan ner e lo si cuoce nei forni tradizionali appositamente restaurati e rimessi in funzione (http://appweb.regione.vda.it). Lo stesso succede in diverse località piemontesi di cui possiedo documentazione rilevata sul terreno.

Ma la segale in ambito alimentare non è solo pane: il malto di segale, che si ricava dalla macerazione delle cariossidi germinate, è usato nella produzione di birra, whisky e vodka $^{11}$. Per il nostro Paese valga l'esempio dell'Ecomuseo della Segale di Sant'Anna di 
Valdieri ( $\mathrm{CN})$, che da qualche tempo promuove la coltivazione della segale per la birra: nell'estate 2012 è stata messa a punto la Brunalpina, prodotta dal birrificio Troll di Vernante (CN) (www.ecomuseosegale.it).

L'interesse verso un ritorno della coltura della segale, oltre all'utilizzo in campo alimentare, ha portato a sperimentare, o in alcuni casi a recuperare, alcuni utilizzi della paglia in ambito edilizio. I tetti delle abitazioni costituiscono forse l'esempio maggiormente rappresentativo: in passato erano diffusi soprattutto nel cuneese, dove la tecnica di costruzione è stata recuperata dal già citato Ecomuseo della Segale. Questa tipologia di tetto è stata sperimentata anche in Val Sangone e dall'associazione torinese Edilpaglia, il cui obiettivo è diffondere sul territorio nazionale le tecniche di costruzione che hanno come base la paglia, tra cui anche quella di segale, e di censire le abitazioni realizzate con questi materiali.

La segale era quasi totalmente scomparsa nel XX secolo nelle Alpi occidentali, con la progressiva conversione dei campi coltivati in prati stabili, a causa dello spopolamento e dell'abbandono delle terre alte e della concorrenza con le coltivazioni meccanizzate della pianura. Una sintetica analisi di iniziative e progetti in atto nell'arco alpino ci permetterà di rispondere a tali quesiti. La ricerca sul campo è stata preceduta da una prima fase di inquadramento teorico dell'indagine e dalla raccolta di documentazione bibliografica per approfondire i contesti di riferimento. Si è compiuta inoltre un'analisi dei bisogni degli attori delle comunità locali. La raccolta dei dati sul campo è avvenuta principalmente tramite interviste videoregistrate con coloro che si sono fatti promotori delle realtà citate di seguito ma anche chiacchierate informali avvenute durante eventi organizzati dalle varie associazioni locali di agricoltori ${ }^{12}$ hanno permesso di conoscere pratiche, intenti, iniziative e contraddizioni in atto nella loro comunità di appartenenza: significativo è che questi informatori privilegiati fossero per lo più persone che ricoprono o hanno ricoperto una funzione sociale e/o culturale rilevante e riconosciuta all'interno del proprio paese e che, di conseguenza, sono risultati essere i più informati, anche per il ruolo sociale appunto acquisito nel tempo all'interno della comunità, e spesso hanno orientato «il nucleo della [...] ricerca durante l'etnografia» (Miller et al. 2018: 36). È stato inoltre ritenuto utile indagare i loro bisogni e reperire informazioni su attività cadute in disuso da parte di persone anziane prima che questo patrimonio di memorie vada perduto. Questo, in un prossimo futuro, permetterà un interessante confronto tra le tecniche e i saperi tradizionali e quelli attuali, così da analizzare evoluzioni e permanenze e ottenere una conoscenza quanto più completa delle varie prassi.

In Valle d'Aosta, in cui ho compiuto la ricerca più recente nei mesi scorsi, sono diversi i progetti in atto che mirano soprattutto alla conservazione della biodiversità, nel recupero del materiale genetico di origine locale e nella caratterizzazione dei diversi ecotipi valdostani di segale. Mauro Bassignana, direttore della sperimentazione dell'Institut Agricole Régional, afferma che nella sua regione fino al 1900 8mila ettari erano coltivati a cereali; dall'inizio del nuovo secolo si registra un rinnovato interesse per le colture cerealicole, in particolare per la segale, ma, nonostante ciò, non sono aumentate le superfici ad essa dedicate, che attualmente si attestano sui 30 ettari, distribuiti per lo più in piccoli appezzamenti mantenuti per il consumo familiare. Esistono però realtà molto interessanti, quali Morgex e La Salle, i due paesi vincitori del premio per il miglior pane nero della regione ${ }^{13}$. Bassignana segnala inoltre che l'associazione coltivatori "Lo Gran" ha cercato di produrre o di dotarsi di una certa meccanizzazione messa a disposizione dei soci, per ovviare alle difficoltà connesse alla 
raccolta nei terreni impervi. Ritengo però significativo notare che i soci di "Lo Gran" coltivano soprattutto varietà svizzere e non locali ${ }^{14}$, diversamente dall'azienda Bonne Vallée di Donnas, che da qualche anno semina segale autoctona valdostana "Arturo", dal nome del contadino che ne ha donato le sementi all'Institut Agricole Régional. Probabilmente questa è proprio la varietà coltivata per secoli sui terrazzamenti e nei fondivalle valdostani (www.bonnevallee.com). Di forte impatto sul territorio è il progetto La Vallée du Seigle - entre fours et moulins attivato nel 2013 dal Comune di Rhêmes-Saint-Georges - con fondi ottenuti dal PSR (Piano di Sviluppo Rurale) e con la collaborazione dell'Institut Agricole Régional ${ }^{15}$ - per valorizzare la filiera dell'ecotipo di segale locale e riscoprire il patrimonio architettonico ${ }^{16}$ e storico-culturale presente nelle frazioni del territorio comunale. Gli informatori dichiarano che qui l'arte panificatoria non è mai stata abbandonata, ritengo quindi opportuno segnalare $i$ prodotti innovativi creati nell'ambito di tale iniziativa. Irene, chef del ristorante Le Barmé de l'Ours, con le sue nuove proposte gastronomiche - dall'antipasto al gelato sostiene di aver inventato un turismo che attrae coloro che vogliono assaporare un prodotto autoctono a km zero; il figlio Andrea pone l'accento sulla birra Blo Emotion, che si vorrebbe commerciare solo nella zona in cui la segale è microfiliera ${ }^{17}$. Elisangela, di origini brasiliane, da dieci anni con il marito alleva bovine valdostane: hanno aderito al progetto perché sono convinti che con il recupero di una coltura automaticamente si rivitalizzi la cultura locale, si crei un'identità del territorio e si costruisca una rete all'interno della comunità.

In Valle d'Aosta esistono alcuni casi di "trasformatori" - come li ha definiti Bassignana -, cioè di panettieri, anzi di biopanettieri, che da tempo panificavano con farine biologiche e che recentemente, consolidando il loro già forte radicamento al territorio, hanno cominciato anche a coltivare piccoli appezzamenti con vari cereali, la segale innanzitutto: questi "nuovi contadini" ${ }^{18}$ scelgono di fare agricoltura su piccola scala recuperando saperi e tecniche legati alla tradizione (Willis, Campbell 2004). Tali iniziative rientrano a pieno titolo nel fenomeno che definiamo neoruralismo, che si fonda sulla percezione delle svariate possibilità dal punto di vista professionale e di modalità dell'abitare (Romita, Núñez 2014). Ritengo interessante riportare la riflessione di Valerio Merlo che distingue il «neoruralismo elitario degli industriali e dei grandi borghesi» degli anni '50-'60 dal «neoruralismo protestatario degli anni Settanta, di cui sono protagonisti i delusi del Sessantotto [...] che vedono nel ritorno all'agricoltura l'unica e l'ultima possibilità di sperimentare praticamente una alternativa al modo di vivere capitalistico», da quello attuale, iniziato nel decennio Ottanta, che "ha come protagonista il cittadino che cerca in campagna, non una alternativa di vita o professionale, bensì una residenza complementare a quella urbana» (2006: 171). Nello specifico, nel contesto che si sta analizzando, il neoruralismo «delinea una situazione di nuove opportunità per le forme di produzione (e consumo)» (Corti 2007: 176). I neocontadini dell'arco alpino sono nuovi abitanti, soprattutto giovani che hanno deciso di trasferirsi in montagna, sostenere qui un progetto di vita e investire su un sistema economico sostenibile: il neoruralismo che si tratteggia assume una valenza sociale, «comportando la ricomposizione di un tessuto rurale che la modernità e contemporaneità parevano aver definitivamente destrutturato e ponendo $i$ presupposti di reti di relazioni e solidarietà urbano-rurali» (Corti 2007: 180).

Per quanto riguarda il Piemonte, ho avuto modo di trattare in altre sedi il recupero della segale (Bonato 2017, 2020): mi limiterò quindi ad alcuni accenni rimandando ai riferimenti indicati per l'approfondimento. Sfumato l'obiettivo di reintrodurre la 
coltivazione della segale a Moncenisio ${ }^{19}$, in Valle Susa - nell'ambito del già citato progetto M.A.S.K.A. -, presente in passato sulle alture della zona, e avviarne così la filiera, il gruppo di ricerca ne ha documentato la persistenza nella vicina frazione Cels, del comune di Exilles (TO). Qui la segale dopo la raccolta viene portata ad un mulino nella bassa Valle e macinata per produrre farina, ma in futuro l'azienda agricola produttrice, "La Vincia", intende ampliare le superfici coltivate, aumentando la riconversione delle terre marginali in aree produttive, in modo da poter disporre di quantità più consistenti per $\mathrm{i}$ consumatori che ne apprezzano l'alto valore nutritivo $\mathrm{e}$ gustativo; inoltre vorrebbe completare la filiera con la possibilità di panificare direttamente le farine prodotte; non da ultimo, un ulteriore obiettivo è rivolto alla produzione di paglia per realizzare i tetti delle abitazioni. E questo era l'iniziale intento dell'Ecomuseo della Segale di Sant'Anna di Valdieri, in Valle Gesso (CN): riattivare la produzione di questo cereale per ottenere la paglia per la copertura dei tetti secondo la tradizione valliva. Il recupero di alcune abitazioni e dei loro tetti in segale "ha permesso l'ampliamento dell'anello escursionistico relativo all'Ecomuseo» (www.ecomuseosegale.it) oltre ad essere stato «funzionale alla ricostruzione della vita quotidiana nell'ambiente domestico e nella stalla, attraverso gli allestimenti interni e con il recupero dei terreni circostanti» (Ibidem). In realtà questa è stata la molla propulsiva per estendere la coltivazione su ampi appezzamenti di terreno e per promuovere la festa della segale, che si svolge dal $1992^{20}$, durante la quale si rievoca la tradizionale battitura del cereale che nelle prime settimane di agosto, nei cortili, coinvolgeva l'intera comunità.

Alcune aziende della Valle Grana $(\mathrm{CN})$ recentemente hanno riproposto la coltivazione del barbariato, il barbarià ${ }^{21}$, un'antica tecnica che prevede la semina autunnale di una miscela composta da semi di grano (60\%) e segale (40\%); è documentata già nel 1480 , quando «il consiglio dell'ospedale di Cuneo delibera di affittare le terre dell'Ente contro la prestazione del canone della metà del frumento raccolto e della terza parte della segale e del barbariato oltre a un'emina aggiuntiva per ogni giornata seminata con quest'ultima mistura» (Panero 1991: 112) ed è stata praticata fino a pochi decenni fa. La coltura del barbariato è stata ripresa nell'ambito del progetto biosostenibile e agrotradizionale della rotazione temporale necessaria (4 anni) per la produzione dell'aglio di Caraglio.

In Lombardia, in particolare in Valle Camonica, la segale, meglio di altri cereali, per condizioni ambientali, si presta al recupero: iniziato con varietà industriali, da quest'anno sono state introdotte quelle locali. Rachele Stendella, agronoma della Rete Semi Rurali ${ }^{22}$, che segue il progetto sui cereali tradizionali per il bio-distretto Valle Camonica, afferma che dal prossimo anno si adotterà la strategia del mescolamento. Il nuovo progetto in atto, che si colloca in continuità con il precedente Coltivare paesaggi resilienti ${ }^{23}$, intende promuovere l'utilizzo della segale preparando pane che contiene il $70-80 \%$ di farina di questo cereale. Stendella spiega che le motivazioni del suo recupero sono culturali e colturali e innanzitutto legate alla tradizione: la significativa richiesta da parte dei turisti di pane e gnocchi, alimenti tipici della zona, fino a poco tempo fa preparati utilizzando segale importata, ha stimolato la coltivazione di un ecotipo locale. Questa iniziativa rimanda al concetto di autenticità - il cui dibattito da tempo anima (soprattutto) gli antropologi - e sembra collocarsi nell'ambito della valorizzazione del patrimonio locale per cui tale nozione è «una categoria consustanziale alla logica patrimoniale e quindi [...] riferimento necessario per attribuire a un determinato elemento la qualifica di bene culturale» (Bortolotto 2011: 7). Per quanto riguarda la 
ricerca di autenticità da parte dei turisti, non è superfluo ricordare che questa è sempre altrove, in altri periodi storici, in altre culture, soprattutto in stili di vita più semplici (MacCannell 1976): in particolare la cultura popolare appare portatrice di autenticità, di verità, in contrapposizione all'artificiosità, alla falsità della cultura di massa (Dei 2002). Nel recupero della coltura della segale in Valle Camonica entrano poi in gioco le questioni agronomiche, perché le aziende che la praticano sono collocate in alto e quindi la segale è più rustica e di conseguenza più rinomata.

Sempre in Lombardia, il progetto "Segale 100\% Valtellina", giunto nel 2021 alla quinta edizione, mira alla reintroduzione e alla valorizzazione di questo cereale e alla produzione di un tipo di pane realizzato con segale esclusivamente locale. Promosso dall'Unione del Commercio e del Turismo e dei Servizi della provincia di Sondrio, quest'anno coinvolge tre coltivatori e sedici panifici (www.segalevaltellina.it). In Alto Adige nel 2011 è stato avviato il progetto "Regiograno" per rilanciare la coltivazione cerealicola creando una rete di collaborazione tra agricoltori, mugnai e panificatori: ad oggi più di 50 aziende coltivano segale in Val Venosta, Val Pusteria, Val d'Isarco e sul monte Zoccolo ${ }^{24}$ (www.idm-suedtirol.com). Nonostante le pratiche in corso e il quadro dinamico che si è delineato, nel nostro paese la segale rappresenta una coltura minoritaria: nel 2020 ha interessato poco più di 3.500 ettari con una produzione di 117.544 quintali (https.dati.istat.it) e il primato della Calabria ${ }^{25}$, dove il consumo di questo cereale si sta diffondendo, tanto che l'Arsac (Agenzia regionale dei servizi in agricoltura) ha proposto l'attivazione di una filiera con indicazione precisa e riconosciuta dell'origine (www.parcoaspromonte.gov.it).

\section{Fruibilità}

Le iniziative qui schematicamente presentate suggeriscono che le pratiche agricole che si stanno riattivando in più parti non sono solo testimonianze del passato ma si propongono come espressione di operatività e di nuove attività. E l'acquisizione delle competenze ad esse relative rivelano oggi una sorta di "paradosso dei saperi":

se in passato il processo di formazione aveva luogo solo oralmente e in senso verticale, dagli anziani ai bambini, tanto da possedere una dimensione temporale estremamente accentuata, oggi la trasmissione è extrafamigliare e si attua anche in senso orizzontale, coinvolgendo persone di età diverse, come i "nuovi abitanti" che riscoprono attuali vecchie professionalità - o addirittura, se è concesso il termine, in senso obliquo, da anziani detentori di saperi locali a giovani neo-abitanti che di queste tradizioni sono disposti, e spesso ansiosi, di farsi portatori (Bonato, Viazzo 2013: 24).

Con il processo di emigrazione dalla montagna verso la pianura e i grossi centri urbani, che prende avvio a partire dal secondo dopoguerra ${ }^{26}$, quasi tutti $i$ terreni precedentemente coltivati sono gradualmente lasciati incolti. Con il loro abbandono si è perso anche quel processo di inculturazione che riguardava conoscenze e saperi di natura orale che avevano caratterizzato fino ad allora la società contadina: ne consegue che le competenze tradizionali oggi vengono in parte acquisite dai "nuovi abitanti" provenienti dalle città o, comunque, da zone extra-montane - le quali si intrecciano a nuovi saperi che, inevitabilmente, interagiscono con i repentini cambiamenti socioculturali, storico-economici e ambientali della nostra epoca. Spesso i nuovi abitanti si insediano in realtà abbandonate creando un nuovo territorio laddove quello precedente si stava perdendo. In geografia questo fenomeno si interpreta attraverso il modello del 
ciclo T-D-R, in cui vi è un'area che è stata formata e plasmata, seguita da una deterritorializzazione con perdita delle funzioni di quel luogo e infine da una riterritorializzazione con la conseguente trasformazione e acquisizione di nuove funzioni per quel territorio. Questo modello può essere applicato per descrivere quanto è accaduto a molte borgate e a tante costruzioni di montagna, abbandonate dopo aver perso la loro funzione legata al lavoro agricolo e oggi recuperate come abitazioni da parte di chi lavora in settori extra agricoli o nel turismo (Pettenati 2009). Ed è quanto emerge nei pochi e sintetici esempi riportati, in cui sembra di individuare la spinta iniziale nel desiderio di rilanciare una produzione artigianale, che poi, a seconda del contesto, ha sviluppato percorsi differenti, se pur, a mio parere, si risolvano in obiettivi condivisi: le motivazioni strettamente economiche che avevano generato l'abbandono della coltura della segale sembrano oggi compensate da quelle di ordine culturale, sociale e ambientale. Mi pare opportuno notare che tali processi "interni" alla comunità sono in costante dialogo con il turismo, che può essere un'occasione di rilancio economico per molte località minori delle Alpi; e il turista stesso, alla ricerca della natura incontaminata e di elementi della tradizione contadina agropastorale, spesso funge da incentivo all'organizzazione di eventi.

Ciò che appare come un recupero della tradizione mette in luce un rinnovato uso del territorio e dimostra di essere una nuova forma di "cultura delle colture", legata indubbiamente alle sue abituali pratiche ma che contemporaneamente cambia la prospettiva dello stile di vita di chi è impegnato nel mestiere di coltivatore stimolando il ritorno alla montagna e incoraggiando progetti di biosostenibilità ambientale. Si consideri che la valorizzazione dei prodotti locali e tipici di un territorio preserva e promuove la biodiversità tutelando al contempo l'ambiente, il cui potenziale produttivo è garantito proprio da una gestione orientata a conservare la biodiversità. Il recupero e/o l'innesto di specie autoctone genera altresì buone pratiche quali l'avvio di una filiera produttiva e lo sviluppo del consumo di prossimità. Gli imprenditori locali e le aziende agricole usano le risorse dei loro territori per incrementare la qualità della vita operando quindi in un'ottica di sostenibilità, concetto che qui, nello specifico, si traduce anche - e soprattutto - nella riconversione di terre marginali in aree produttive: reinvestire nel "capitale naturale", usare in maniera responsabile le risorse della terra, recuperare ecotipi locali, limitare l'impatto ambientale, sostenere l'azione sul paesaggio sono solo alcuni dei modi in cui la sostenibilità può essere raggiunta ${ }^{27}$. Quella che si pratica è indubbiamente un'agricoltura di qualità - e una conseguente microeconomia sostenibile che produce reddito - che si traduce in una opportunità di sviluppo e in un efficace e consapevole strumento di tutela del paesaggio: la mia esperienza sul campo mi permette di affermare che genera inoltre coesione sociale, la quale determina processi di sperimentazione dal basso e nuovi orizzonti di senso all'interno delle comunità, che in alcuni casi assumono un ruolo fattivo nell'impostazione di politiche territoriali ${ }^{28}$.

Un'altra positiva ricaduta della salvaguardia e della promozione delle tradizioni è l'organizzazione di eventi che ripropongono la cultura locale, preindustriale, contadina e artigianale unitamente a feste "inventate" legate a mestieri e pratiche del passato che, «oltre ad essere un luogo di incontro, confronto, collaborazione, progettualità tra $\mathrm{i}$ diversi soggetti dell'imprenditoria locale, offre a questi stessi l'occasione per aprirsi all'esterno» (Bonato 2020: 57). Queste iniziative, che coniugano in maniera egregia sviluppo e sostenibilità, stanno stimolando la nascita di un turismo sostenibile, responsabile e soprattutto esperienziale, che offre esperienze e attività nel luogo in cui 
si soggiorna che trasmettono a chi ne usufruisce l'autenticità del territorio, le sue tradizioni:

è un evento vissuto in prima persona che produce un effetto sui nostri sensi e lascia un segno nel nostro vissuto. E vuole proporre attività immersive che siano uniche ma che possano anche influire con modalità sostenibili e responsabili alla valorizzazione del territorio. L'esperienza, quindi, non è vissuta solo da chi la fruisce ma anche da chi la eroga (Bonato 2020: 132).

Le azioni intraprese e i programmi avviati finora nelle aree marginali mostrano di essere adatte allo sviluppo di progetti innovativi fondati sul rispetto per l'ambiente e sulla valorizzazione dei siti in abbandono, in sintonia con l'adesione sempre più significativa delle realtà locali alle politiche ambientali internazionali e alle tante strategie virtuose che queste mettono in atto. Con convinzione i promotori riescono a coniugare in maniera egregia sviluppo e sostenibilità: perché nei contesti indagati non è equivoco e incoerente uno sviluppo sostenibile? Si è qui delineata una situazione «di nuove opportunità per le forme di produzione (e consumo)»(Corti 2007: 176) ma soprattutto sono stati presentati territori dinamici, resi tali da comunità collaborative che, operando per una gestione il più possibile sostenibile delle risorse locali, contribuiscono all'elaborazione e alla crescita del proprio patrimonio culturale e al contempo presentano all'esterno il loro senso di appartenenza, la propria specifica cultura. E la tradizione è il linguaggio con cui comunicano.

Ritengo che l'osservazione e la documentazione del ritorno alle colture abbandonate in territorio alpino necessitino di un incoraggiamento non tanto in senso produttivistico quanto, piuttosto, in funzione del mantenimento del paesaggio culturale. Nuovi imprevisti e allarmanti scenari richiamano la nostra attenzione: pascoli, terreni e boschi non più curati sono ora un'emergenza ambientale e culturale che i diversi saperi disciplinari, in sinergia, possono aiutare ad arginare attraverso il dialogo aperto con gli attori locali, pubblici e privati. Queste tematiche possono rientrare in un dibattito più ampio e trasversale che comprende nella critica sia le proprie epistemologie occidentali sia quelle dei diversi ambientalismi e che stimola la comprensione e l'analisi delle risposte e delle iniziative comunitarie a problemi quali il disboscamento, le alluvioni, i cambiamenti climatici, la costruzione delle grandi opere infrastrutturali, il consumo del suolo, l'uso dell'acqua, così come l'indagine sui processi di sperimentazione dal basso e sui nuovi orizzonti di senso all'interno dei movimenti sociali, l'autorganizzazione della produzione e del consumo.

\section{BIBLIOGRAFIA}

Annunziata A., 2011 «Il ruolo del territorio come elemento di valorizzazione delle produzioni agroalimentari: il caso dei prodotti tipici e locali», in A. Cesaretti, A. Annunziata (a cura di), Strategie e strumenti per la valorizzazione sostenibile delle produzioni agroalimentari di qualità, FrancoAngeli, Milano: 107-127.

Barger G., 1931 Ergot and Ergotism, Gurney and Jackson, London. 
Bätzing W., 2002 I processi di trasformazioni di ambiente, economia, società e popolazione attualmente in corso nelle Alpi, Bundesministerium für Umwelt, Naturschutz und Reaktorsicherheit, Berlin.

Besson-Girard J.-C., 2007 Decrescendo cantabile: piccolo manuale per una decrescita armonica, Jaca Book, Milano; trad. it. di Decrescendo cantabile. Petit manuel pour une décroissance harmonique, Parangon, Lyon, 2005.

Bonato L., 2017 «Fra abbandoni e ritorni: aree marginali, terre originali», in L. Bonato (a cura di), Aree Marginali. Sostenibilità e saper fare nelle Alpi, FrancoAngeli, Milano: 7-23.

Bonato L., 2020 «Ritualità d'alta quota, tra politiche culturali e sostenibilità», in EtnoAntropologia, 2: 51-70.

Bonato L., 2020 «Turismo nelle terre alte: comunità, politiche culturali e strategie sostenibili», in L. Bonato, D. Cortese, E. Lusso, C. Trinchero (a cura di), Open tourism. Ricerche, prospettive e letture sul turismo culturale nell'area alpina occidentale, Centro Internazionale di studi sugli Insediamenti Medievali, Cherasco: 131-144.

Bonato L., Viazzo P.P., 2013 «www.tradizione.it (sito in costruzione): nuove sfide per l'antropologia alpina», in L. Bonato, P.P. Viazzo (a cura di), Antropologia e beni culturali nelle Alpi. Studiare, valorizzare, restituire, Edizioni dell'Orso, Alessandria: 9-27.

Bortolotto C., 2011 «Patrimonio immateriale e autenticità: una relazione indissolubile», in La Ricerca Folklorica, 64: 7-17.

Breda N., 2013 «La montagna vista dalla pianura. Implicazioni per la montagna, per la pianura e per l'antropologia», in L. Bonato, P.P. Viazzo (a cura di), Antropologia e beni culturali nelle Alpi. Studiare, valorizzare, restituire, Edizioni dell'Orso, Alessandria: 55-68.

Caporael L.R., 1976 «Ergotism: The Satan Loosed in Salem?», in Science, 192: 21-26.

Colajanni A., 2008 «Note sulla sostenibilità culturale dei progetti di sviluppo», in F. Zanotelli, F. Lenzi Grillini (a cura di), Subire la cooperazione?, ed.it, Catania.

Colajanni A., Giancristofaro L., Sacco V., 2020 Le Nazioni Unite e l'antropologia. La dimensione culturale nei programmi dell'UNESCO, della Banca Mondiale, della FAO e dell'IFAD, CISU, Roma.

Corti M., 2007 «Quale neoruralismo», in L'Ecologist, 7: 168-189.

Dei F., 2002 Beethoven e le mondine. Ripensare la cultura popolare, Meltemi, Roma.

Ginzburg C., 1989 Storia notturna. Una decifrazione del sabba, Einaudi, Torino.

Grasseni C., 2013 Beyond Alternative Food Networks: Italy's Solidarity Purchase Groups, Bloomsbury Academic, London.

Lockyer J., Veteto J.R., 2013 Environmental Anthropology Engaging Ecotopia. An Introduction, in J. Lockyer, J.R. Veteto (eds.), Environmental Anthropology. Engaging Ecotopia. Bioregionalism, Permaculture, and Ecovillages, Berghahn, New York/Oxford: 1-31.

Latouche S., 2008 La scommessa della decrescita, Feltrinelli, Milano; trad. it. di Le pari de la décroissance, Fayard, Paris, 2006.

MacCannell D., 1976 The Tourist: A New Theory of the Leisure Class, Schocken Books, New York.

Mancini R., 2015 Ripensare la sostenibilità. Le conseguenze economiche della democrazia, FrancoAngeli, Milano.

Matonti L., 2015 Erbe e antichi rimedi di ieri, oggi e domani, Il Graffio, Borgone Susa.

Merlo V., 2006 Voglia di campagna. Neoruralismo e città, Città Aperta, Troni (EN). 
Miller D. et al., 2018 Come il mondo ha cambiato i social media, Ledizioni, Milano.

Nada Patrone A.M., 1981 Il cibo del ricco ed il cibo del povero. Contributo alla storia qualitativa dell'alimentazione. L'area pedemontana degli ultimi secoli del Medio Evo, Centro Studi Piemontese, Torino.

Orlando G., 2018 «Le reti alternative del cibo dopo la crisi. Teoria, ipotesi di lavoro e un caso studio esplorativo», in Archivio Antropologico Mediterraneo, 20.

Panero F., 1991 «Viticoltura, atti mezzadrili e colonia parziaria nel Piemonte centro-meridionale (secolo XV-XVI)», in R. Comba (a cura di), Vigne e viti nel Piemonte rinascimentale, L'Arciere, Cuneo: 105-129.

Pérez-Vitoria S., 2007 Il ritorno dei contadini, Jaca Book, Milano.

Pettenati G., 2010 «I nuovi abitanti di Stroppo (Val Maira). Riflessioni sul nuovo popolamento di un comune alpino», in F. Corrado, V. Porcellana (a cura di), Alpi e ricerca. Proposte e progetti per $i$ territori alpini, FrancoAngeli, Milano: 133-145.

Porcellana V., Stefani S., 2016 «Processi partecipativi e pratiche collaborative per progettare il futuro», in V. Porcellana, S. Stefani (a cura di), Processi partecipativi ed etnografia collaborativa nelle Alpi e altrove, Edizioni dell'Orso, Alessandria: 7-22.

Rappoport L., 2003 Come mangiamo. Appetito cultura e psicologia del cibo, Ponte alle Grazie, Milano; trad. it. di How We Eat: Appetite, Culture, and the Psychology of Food, ECW Press, Toronto, 2003.

Romita T., Núñez S., 2014 «Nuevas poblaciones rurales: rural users, trashumantes, nuevos habitantes new rural population: rural users, nomadic and new inhabitants», in Gran Tour: Revista de Investigaciones Turísticas, 10: 4-22.

Salsa A., 2007 Il tramonto delle identità tradizionali: spaesamento e disagio esistenziale nelle Alpi, Priuli \& Verlucca, Scarmagno (TO).

Saltini A., 2010 I semi della civiltà. Frumento, riso e mais nella storia delle società umane, Nuova Terra Antica, Milano.

Sassoon D., 2006 The culture of Europeans, Harper Collins, London.

Siniscalchi V., 2009 «I processi di tipicizzazione tra singolarità e ripetizione», in Culture della sostenibilità, 6: 51-64.

Tassan M., 2017 «Cibo "naturale" e food activism. Il consumo critico in due Gruppi di Acquisto Solidale nell'area Milanese», in Archivio Antropologico Mediterraneo, 19: 23-31.

Van der Ploeg J.D., 2009 I nuovi contadini. Le campagne e le risposte alla globalizzazione, Donzelli, Roma, trad. it. di The New Peasantries. Struggles for Autonomy and Sustainability in an Era of Empire and Globalization Earthscan, Sterling, London, 2008.

Viazzo P.P., 2014 «Nuovi montanari», in Antropologia Museale, 34-36: 107-109.

Willis S., Campbell H., 2004 «The Chestnut Economy: The Praxis of Neo-Peasantry in Rural France», in Sociologia Ruralis, 44(3): 317-331.

Wong J.G., 1998 «Ergot of Rye: History», in Botany, 135.

Sitografia

http://appweb.regione.vda.it [ultima consultazione in data 10 maggio 2021] 
$100 \%$ Segale Valtellina

www.segalevaltellina.it [ultima consultazione in data 14 maggio 2021]

Atomik, la prima vodka prodotta con il grano contaminato di Chernobyl

www.repubblica.it [ultima consultazione in data 12 maggio 2021]

Bio, km zero e made in Italy: cresce l'attenzione alla sostenibilità della spesa

www.ilsole24ore.com [ultima consultazione in data 10 maggio 2021]

Carovana delle Alpi

www.legambiente.it [ultima consultazione in data 12 luglio 2021]

Coltivare paesaggi resilienti

www.biodistrettovallecamonica.it [ultima consultazione in data 14 maggio 2021]

Dalla terra alla tavola

www.bonnevallee.com [ultima consultazione in data 10 maggio 2021]

Il Regiograno dell'Alto Adige spegne dieci candeline

www.idm-suedtirol.com [ultima consultazione in data 23 giugno 2021]

La segale. Buona per la pancia, buona per la stalla e buona per le case

www.ecomuseosegale.it [ultima consultazione in data 2 maggio 2021]

Rete Semi Rurali

www.semirurali.net

Segale il cereale rustico dei parchi nazionali calabresi

www.parcoaspromonte.gov.it [ultima consultazione in data 25 giugno 2021]

\section{NOTE}

1. Traduzione del proverbio piemontese A l'è mac le muntagne c'a s'ancuntru nen.

2. Nel movimento neorurale rientrano anche i figli di agricoltori che scelgono di continuare l'attività dei genitori.

3. Interessante la posizione dello storico inglese Donald Sassoon, autore di The culture of Europeans (2006), che reputa la cultura un oggetto commerciale, che viene venduto e comperato.

4. Si veda a tal proposito la Convenzione UNESCO, che già nel 2005 identificava nella cultura un elemento fondamentale per la sostenibilità dello sviluppo e per l'affermarsi di società pacifiche, inclusive e rispettose delle diversità culturali.

5. Segnalo a tal proposito l'esistenza e l'impegno dei “custodi dei semi", ovvero gruppi di agricoltori che prendono in affidamento dei semi e li riproducono nei propri terreni allo scopo poi di distribuirne una parte ad altri coltivatori, mettendo in moto un circolo virtuoso di produzione di prodotti locali per non perdere certe varietà. Per quanto riguarda il territorio piemontese ho avuto modo di confrontarmi con l'associazione "Princìpi Pellegrini DiVangAzioni", il cui scopo è sostenere, sviluppare e riportare la biodiversità che si è persa a causa dell'agricoltura di massa e intensiva, che ha promosso un'iniziativa denominata PASO (Progetto Autoproduzione Sementi Ortive) con la creazione di un gruppo di "custodi" e sta sperimentando la coltura della segale.

6. Questo cereale probabilmente proviene dall'Asia sud-occidentale, dove cresceva spontaneamente come erbaccia nei campi di frumento e orzo; inizialmente destinata al nutrimento del bestiame, è stata addomesticata intorno all'anno 1.000 a.C. e impiegata nella panificazione (Saltini 2010). Da quel momento cominciano a farne uso anche Celti, Germani e Liguri (Wong 1998). 
7. Alcune specie di questo fungo contengono un alcaloide denominato ergonovina dal quale nel 1943 è stato sintetizzato in laboratorio l'acido lisergico dietilamide (LSD) (Ginzburg 1989).

8. Attraverso l'ingestione di segale cornuta Ginzburg fornisce un'interpretazione della complessa esperienza del sabba stregonesco, pur puntualizzando che la sua ripetizione codificata può essere solo culturale: «nessuna forma di privazione, nessuna sostanza, nessuna tecnica estatica» (1989: 284) può sollecitarla. Caporael (1976) ha ipotizzato che gli accadimenti verificatisi a Salem nel 1692 e interpretati allora come possessione diabolica fossero in realtà episodi di ergotismo convulsivo.

9. La segale possiede proprietà energetiche, depurative e ricostituenti e protettive per il sistema cardiovascolare, per l'intestino; previene l'invecchiamento ed è ottima per la salute della pelle. Rende il sangue fluido e combatte l'arteriosclerosi; favorisce la peristalsi intestinale e aumenta la potenza muscolare (Matonti 2015).

10. Questo manufatto consta di diversi pannelli montati sulle ante di una libreria e ha una lunghezza complessiva di più di 2 metri. La direttrice del Centre, Christiane Dunoyer, mi ha detto che l'opera è stata realizzata da un artista locale, Gino Thomasset, e collocata nel locale al momento della sua inaugurazione, nel 1987.

11. Il rye whiskey è una bevanda tipicamente nordamericana; la vodka di segale viene prodotta in Polonia e in Russia. È del 2020 la notizia relativa ad una nuova vodka - Atomik - prodotta con la segale di un campo coltivato non lontano dall'ex centrale nucleare di Chernoyl. Si tratta del primo prodotto alimentare proveniente dalla zona di esclusione abbandonata dal 26 aprile 1986. Il progetto, promosso da alcuni ricercatori britannici, intende aiutare l'economia delle zone circostanti l'ex centrale nucleare (www.repubblica.it).

12. Cito a titolo esemplificativo quelli denominati "Ingraniamo", organizzati dall'Associazione "Princípi Pellegrini DiVangAzioni" attiva in Valle Susa (TO), durante i quali agricoltori e semplici appassionati condividevano le competenze acquisite e trasmettevano i loro saperi su come coltivare in territorio montano le antiche varietà di grano, orzo, segale e mais e per scambiarsi le sementi di queste biodiversità.

13. Il concorso, transfrontaliero, promosso dalla Regione Valle d'Aosta, la Regione Lombardia e la Val Poschiavo del cantone svizzero dei Grigioni, "Lo pan ner - I pani delle Alpi”, è stato ideato nel 2016, al termine di un progetto strategico Italia/Svizzera per la valorizzazione del patrimonio culturale immateriale. Questa iniziativa riporta in attività i forni consortili o comunali delle frazioni (http://appweb.regione.vda.it).

14. Nelle future discese sul campo mi riprometto di cercare di capire se l'utilizzo di varietà svizzere è percepito dai coltivatori esterni all'associazione come una sorta di intrusione o se gli aspetti simbolici legati al recupero della coltura sono sufficienti a legittimare tale adozione.

15. Più recentemente il Comune e l'Institut, con il progetto PROBIODIV, iniziato nel 2019 con la collaborazione del Parco Nazionale Gran Paradiso, hanno recuperato un'area incolta con la messa a dimora della segale e, a rotazione, di altre colture di montagna. La sindaca di Rhêmes-SaintGeorges, Nella Therisod, specifica che quest'area è destinata ad una funzione didatticodivulgativa di interesse sia scientifico sia di conoscenza del mondo agricolo cerealicolo.

16. Nello specifico si tratta di 7 forni e 4 mulini visibili oggi nei differenti stati di conservazione, cioè restaurati o nella loro forma originaria. Nell'ambito del progetto questi siti sono stati collegati mediante una proposta di itinerari di visita.

17. La microfiliera, o filiera corta, è una catena produttiva che comporta pochi passaggi, iniziando dalle materie prime per terminare con il consumatore.

18. Van der Ploeg (2009) dimostra che oggi, «dietro l'invisibilità costruita ad arte, ampiamente rafforzata dalla connotazione negativa che il termine contadino ha nel linguaggio quotidiano, esiste una realtà empirica in cui ci sono molti più contadini di quanti ne siano mai esistiti in passato» (2009: 4). I contadini sono una fondamentale opportunità per le società perché sembrano rispondere ai fabbisogni alimentari mondiali nell'ottica e di uno sviluppo sostenibile 
dell'agricoltura e delle economie rurali. Sia nei paesi industrializzati sia in quelli in via di sviluppo si registrano fenomeni complessi di ritorno al mondo contadino i cui protagonisti si configurano non solo come produttori di cibo ma anche quali costruttori di paesaggi e custodi della Terra. E il fulcro di questo modello secondo Van der Ploeg (2009) e Pérez-Vitoria (2007) è la ricerca dell'autonomia rispetto al potere ordinatore degli imperi agroalimentari: entrambi gli studiosi stimolano la ricerca antropologica verso i "nuovi contadini” in un'ottica che tenga conto «della globalizzazione e dei suoi riflussi, [...] della crisi mondiale del capitalismo finanziario e delle rivoluzioni che esso induce, e delle narrazioni che la classe egemone sta producendo» (Breda 2013: 62).

19. Moncenisio è il più piccolo comune del Piemonte e il terzo meno popoloso d'Italia: conta 40 residenti anagrafici.

20. Dal 20 al 22 agosto 2021, per la trentesima edizione della festa, è stato predisposto un programma consono alle disposizioni relative al Covid-19.

21. Letteralmente significa imbarbarito, imbastardito; questo metodo veniva usato dai contadini delle zone montane e pedemontane per ottenere una farina più digeribile rispetto a quella di sola segale e garantiva la sopravvivenza in caso di annate non propizie dal punto di vista climatico per la coltivazione del grano (Nada Patrone 1981).

22. Impegnata nella tutela e nella promozione della biodiversità agricola, la Rete è stata fondata nel 2007 e comprende 47 associazioni (www.semirurali.net).

23. Questo progetto (2018) ha rilanciato la filiera di cereali in Valle Camonica e nel 2021 la rete di agricoltori del bio-distretto ha iniziato a sperimentare vecchie varietà, tra cui la segale, particolarmente adatte all'agricoltura biologica e a basso impatto, tipica delle aree marginali, oltre a distinguersi per le qualità nutrizionali (www.biodistrettovallecamonica.it).

24. Questo territorio vanta una lunga tradizione nella coltivazione di cereali, che a metà del secolo scorso si estendeva su una superficie di circa 30mila ettari, definendosi come parte integrante dell'agricoltura locale. Attualmente l'economia di mercato, rivolta in particolare ai settori frutticolo e lattiero, ha ridotto tale coltivazione a soli 243 ettari (www.idm-suedtirol.com). 25. In questa regione la segale si è ben adattata nei territori sopra ai 750 metri, in particolare in quelli compresi nei tre parchi nazionali (Pollino, Sila, Aspromonte) (www.arsac.calabria.it).

26. Se lo spopolamento alpino si era manifestato in Europa già a partire dal XIX secolo, anche se con tempi e modalità differenti a seconda delle aree, nel nostro paese gli anni maggiormente interessati da questo fenomeno sono quelli compresi tra il 1961 e il 1971 (Bätzing 2002).

27. Mi sembra interessante segnalare l'iniziativa di Legambiente denominata "Carovana delle Alpi" che dal 2002 assegna la bandiera verde alle pratiche innovative e alle esperienze di qualità ambientale e culturale dei territori ad alta quota: nel 2021 ne sono state attribuite 18, tra cui diverse a giovani imprenditori e imprenditrici che puntano su tecnologie e valorizzazione dell'esistente nel ritorno in terra d'origine (www.legambiente.it).

28. La ricerca compiuta nell'ambito del progetto M.A.S.K.A. ha documentato forme di associazionismo legate ai temi dell'agricoltura e della sostenibilità, in particolare le attività dell'Associazione "Principi Pellegrini DiVangAzioni" e i progetti "Ingraniamo" e PASO (Progetto Autoproduzione Sementi Ortive), il movimento "Genuino Clandestino" e l'Associazione "Etinomia". Si tratta, in qualche caso, di associazioni che contano al loro interno persone giovani o "nuovi abitanti" che hanno scelto di trasferirsi in montagna: certamente il loro apporto numerico è ridotto ma risulta rilevante sul piano socio-culturale ed economico. A indagine conclusa si è inoltre rilevata la nascita di fattive collaborazioni tra le realtà già esistenti e l'avvio di una rete - la cui costituzione era uno degli obiettivi del progetto - che le mette in comunicazione sia tra di loro, in modo che condividano e facciano fruttare il loro patrimonio di conoscenze e pratiche, sia con l'esterno (Bonato 2017). 


\section{RIASSUNTI}

Le aree alpine negli ultimi decenni del Novecento sono state testimoni di un notevole processo di spopolamento: gli effetti sull'ambiente causati dall'abbandono delle pratiche agropastorali si sono estesi oltre la dimensione locale, cambiando i caratteri del paesaggio e le tradizioni culturali e trasformando queste aree in terre marginali. Ma da qualche anno nell'intero arco alpino esistono diverse realtà che stanno cercando di reintrodurre differenti tipi di coltivazioni storicamente documentate: ad esempio la segale, cereale di montagna per eccellenza che, pur non essendo originaria delle Alpi, era indispensabile e fondamentale per l'alimentazione e per la quotidianità fino agli anni ' 50 del secolo scorso. Il suo recupero, che è al contempo economico, culturale e turistico, gioca un ruolo significativo - e fortificante, a mio parere - nell'interazione, nella collaborazione e negli scambi tra le comunità locali e funziona da trait d'union fra tradizione e contemporaneità. Questo esempio sarà utile a mostrare che la reintroduzione di coltivazioni locali e storicamente documentate, se rivitalizza l'economia regionale e al contempo tutela l'ambiente, permette di porre l'accento sul fatto che indagare lo spazio alpino attraverso l'implementazione di modelli di sostenibilità culturale ed economica, enfatizzando la rete relazionale e rafforzando l'integrazione e il comportamento attraverso la conoscenzaapprendimento-ricordo, coinvolge le comunità locali in un ruolo più attivo nella società.

In the last decades of the 20th century, the Alpine areas have witnessed a remarkable process of depopulation: the effects on the environment caused by the abandonment of agro-pastoral practices have extended beyond the local scale, changing the characteristics of the landscape and cultural traditions and transforming these areas into marginal lands. For some years now, however, there have been a number of projects throughout the Alps that are attempting to reintroduce different types of historically documented crops: for example, rye, a mountain cereal par excellence which, although not native to the Alps, was essential for food and everyday life until the 1950s. Its recovery, which is at once economic, cultural and touristic, plays a significant - and, in my opinion, fortifying - role in interaction, cooperation and exchanges between local communities and acts as a link between tradition and the present day. This example will be useful to show that the reintroduction of local and historically documented crops, if it revitalises the regional economy and at the same time protects the environment, will allow us to emphasise that investigating the Alpine space through the implementation of models of cultural and economic sustainability, emphasising the relational network and strengthening integration and behaviour through knowledge-learning-remembering, involves local communities in a more active role in society.

\section{INDICE}

Parole chiave : sostenibilità, biodiversità, comunità locale, rivitalizzazione, cultura imprenditoriale

Keywords : sustainability, biodiversity, local community, revitalization, entrepreneurial culture 


\section{AUTORE}

LAURA BONATO

Dipartimento di Lingue e Letterature Straniere e Culture Moderne, Università di Torino laura.bonato@unito.it 